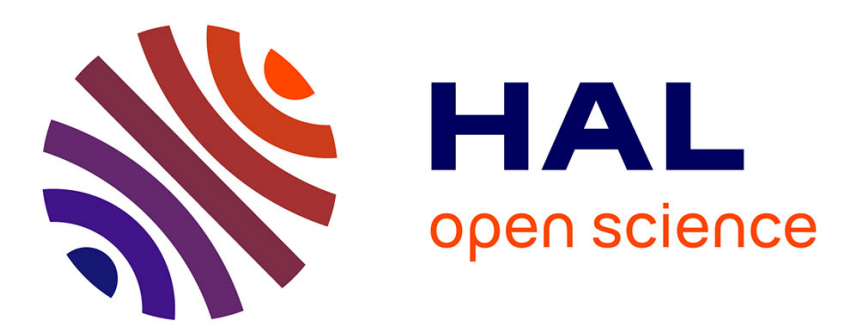

\title{
Fluence Threshold for Photothermal Bubble Generation Using Plasmonic Nanoparticles
}

Khaled Metwally, Serge Mensah, Guillaume Baffou

\section{To cite this version:}

Khaled Metwally, Serge Mensah, Guillaume Baffou. Fluence Threshold for Photothermal Bubble Generation Using Plasmonic Nanoparticles. Journal of Physical Chemistry C, 2015, 119 (51), 10.1021/acs.jpcc.5b09903 . hal-01323089

\section{HAL Id: hal-01323089 \\ https://hal.science/hal-01323089}

Submitted on 30 May 2016

HAL is a multi-disciplinary open access archive for the deposit and dissemination of scientific research documents, whether they are published or not. The documents may come from teaching and research institutions in France or abroad, or from public or private research centers.
L'archive ouverte pluridisciplinaire HAL, est destinée au dépôt et à la diffusion de documents scientifiques de niveau recherche, publiés ou non, émanant des établissements d'enseignement et de recherche français ou étrangers, des laboratoires publics ou privés. 
This document is confidential and is proprietary to the American Chemical Society and its authors. Do not copy or disclose without written permission. If you have received this item in error, notify the sender and delete all copies.

\section{Fluence Threshold for Photothermal Bubble Generation Using Plasmonic Nanoparticles}

\begin{tabular}{|r|l|}
\hline Journal: & The Journal of Physical Chemistry \\
\hline Manuscript ID & jp-2015-09903e.R1 \\
\hline Manuscript Type: & Article \\
\hline Date Submitted by the Author: & n/a \\
\hline Complete List of Authors: & $\begin{array}{l}\text { Metwally, Khaled; Laboratoire de Mécanique et d'Acoustique, UPR 7051 } \\
\text { Mensah, Serge; Laboratoire de Mécanique et d'Acoustique, UPR 7051 } \\
\text { Baffou, Guillaume; Institut Fresnel, Mosaic group }\end{array}$ \\
\hline
\end{tabular}

SCHOLARONE

Manuscripts 


\title{
Fluence Threshold for Photothermal Bubble
}

\section{Generation Using Plasmonic Nanoparticles}

\author{
Khaled Metwally, ${ }^{\dagger}$ Serge Mensah, ${ }^{\dagger}$ and Guillaume Baffou ${ }^{*, \dagger}$ \\ Laboratoire de Mécanique et d'Acoustique, UPR 7051, CNRS, Aix Marseille Université, Ecole \\ Centrale Marseille, 13013 Marseille, and Institut Fresnel, UMR 7249, CNRS, Aix Marseille \\ Université, Ecole Centrale Marseille, 13013 Marseille, France \\ E-mail: guillaume.baffou@fresnel.fr
}

\begin{abstract}
Under nano- to femtosecond pulsed illumination at their plasmonic resonance wavelength, metal nanoparticles efficiently absorb the incident light energy that is subsequently converted into heat. In a liquid environment, with sufficiently high pulse fluences (light energy per unit area), this heat generation may result in the local formation of a transient nanobubble. This phenomenon has been the subject of a decade of investigations and is at the basis of numerous applications from cancer therapy to photoacoutic imaging. The aim of this article is to clarify the question of the fluence threshold required for bubble formation. Using a Runge-Kutta-4 numerical algorithm modeling the heat diffusion around a spherical gold nanoparticle, we numerically investigate the influence of the nanoparticle diameter, pulse duration (from the femto- to the nanosecond range), wavelength and Kapitza resistivity in order to explain the observations reported in the literature.
\end{abstract}

\footnotetext{
${ }^{*}$ To whom correspondence should be addressed

†Laboratoire de Mécanique et d'Acoustique, UPR 7051, CNRS, Aix Marseille Université, Ecole Centrale Marseille, 13013 Marseille

${ }^{\ddagger}$ Institut Fresnel, UMR 7249, CNRS, Aix Marseille Université, Ecole Centrale Marseille, 13013 Marseille, France
} 


\section{Introduction}

Most applications in nanoplasmonics are based on the use of nanoparticles (NPs) in a liquid medium. The presence of a liquid makes the related physics extremely rich. In particular, singular physical processes such as boiling, ${ }^{1-3}$ fluid convection, ${ }^{4,5}$ chemical reaction ${ }^{6}$ or thermophoresis ${ }^{7,8}$ may occur as soon as a plasmonic NP under illumination behaves as a nanosource of heat. The area of research based on the use of plasmonic NPs as heat nanosources is termed thermoplasmonics. ${ }^{9}$

Bubble formation induced by plasmonic heating has been the subject of a decade of investigations, mainly under pulsed illumination ${ }^{10,11}$ (the interest for continuous wave illumination ${ }^{3,12-15}$ is paradoxically much more recent). After the absorption of a pulse of light, the temperature of the NP rises, and subsequently decreases upon releasing heat in the surrounding fluid. If the maximum temperature achieved in the fluid during this process is sufficiently high, bubble formation occurs, a bubble that subsequently collapses on the nanosecond time scale. Such a process has been studied in the context of applications such as photothermal cancer therapy ${ }^{11,16}$ and photoacoustic imaging. ${ }^{17}$

Regarding the underlying physics of bubble generation in plasmonics, three main questions have been arousing the community's interest so far: (i) what is the bubble life time?, (ii) what is the maximum bubble size? and (iii) what is the fluence threshold (light energy per unit area) required for bubble formation (the subject of this article)? Albeit the simplicity of these questions, experimental investigations of nanobubbles are complicated for two reasons. ${ }^{18-21}$ First, due to their nanometric size, they cannot be easily visualized. Even though they can efficiently scatter light and be detectable for this reason, it is hard to directly measure their diameter using optical means due to the diffraction limit. Second, their dynamics lies in the picosecond to nanosecond time scale, which is way below the detection speed of many common detectors in optics.

The fluence threshold for bubble generation in plasmonics has been discussed in several articles, both experimentally and numerically. 1,1,19,21,22 In particular, the overall dependence of the fluence threshold as a function of the NP radius was shown to display a bathtub shape as represented in the following scheme, no matter the pulse duration. $1,19,21$

However, the origin of this non-monotonic profile has not been fully elucidated in the literature. As stated by Hashimito and coworkers in 2012, ${ }^{23}$ the mechanisms of laser-induced bubble formation assisted 


\section{Theoretical framework}

\section{Heat generation in the nanoparticle}

We consider in this work a spherical gold NP, immersed in a uniform liquid environment and illuminated by a single light pulse. The interaction between the incoming light pulse and the NP consists of a three-step process. ${ }^{24}$ (i) Part of the incoming light energy is primarily absorbed by the electronic gas of the metal NP. (ii) This energy is transferred to the atomic lattice of the NP via electron-phonon interaction. (iii) The NP cools down by releasing it thermal energy into the surrounding fluid by thermal diffusion. In this scenario, three time scales are involved: 
1. The pulse duration $\tau_{\mathrm{p}}$. If one considers a Gaussian pulse profile, one can define the pulse duration $\tau_{\mathrm{p}}$ such that the normalized pulse profile reads:

$$
q(t)=\frac{1}{\tau_{\mathrm{p}}} \exp \left(-\pi \frac{\left(t-t_{0}\right)^{2}}{\tau_{\mathrm{p}}^{2}}\right)
$$

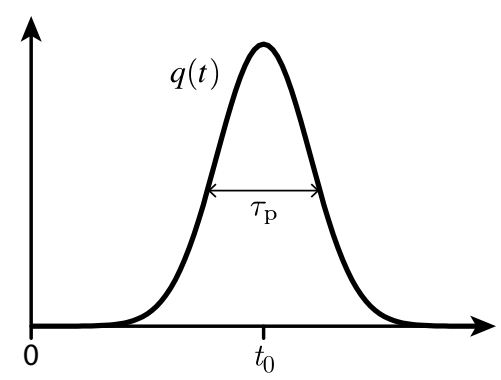

2. The time constant of electron-photon interaction $\tau_{\mathrm{ep}}$. For gold, $\tau_{\mathrm{ep}}=1.7 \mathrm{ps}^{25-27}$ With a good approximation, the power transfer from the electrons to phonons occurs according to an exponential decay function that reads:

$$
p(t)=\frac{1}{\tau_{\mathrm{ep}}} \exp \left(-\frac{t}{\tau_{\mathrm{ep}}}\right)
$$

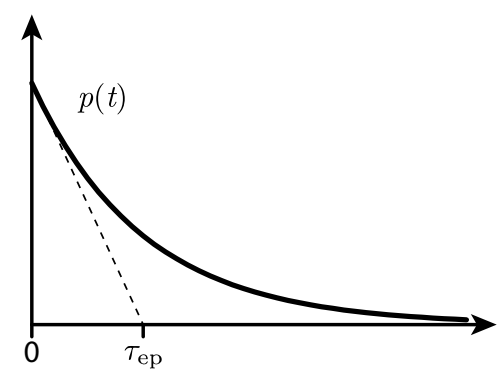

3. The characteristic time of nanoparticle cooling $\tau_{\mathrm{d}}$ due to heat release in the surrounding water. Its expression reads 24

$$
\tau_{\mathrm{d}}=\frac{c_{\mathrm{m}} a^{2}}{3 \kappa_{\mathrm{w}}}
$$

where $a$ is the radius of the NP, $c_{\mathrm{m}}$ is the volumetric heat capacity of the metal $\left(2.5 \times 10^{6} \mathrm{~J} \mathrm{~m}^{-3} \mathrm{~K}^{-1}\right.$ for gold) and $\kappa_{\mathrm{W}}$ is the thermal conductivity of the surrounding water $\left(\kappa_{\mathrm{W}}=0.6 \mathrm{~W} \mathrm{~m} \mathrm{~K}^{-1} \mathrm{~K}^{-1}\right)$. For common NP sizes, it ranges from 1 ps to 1 ns (Fig.1). Note that this time scale may no longer be the characteristic time of NP cooling if a metal/liquid thermal resistance is present at the NP interface, 
as discussed further on (see eq (15)).

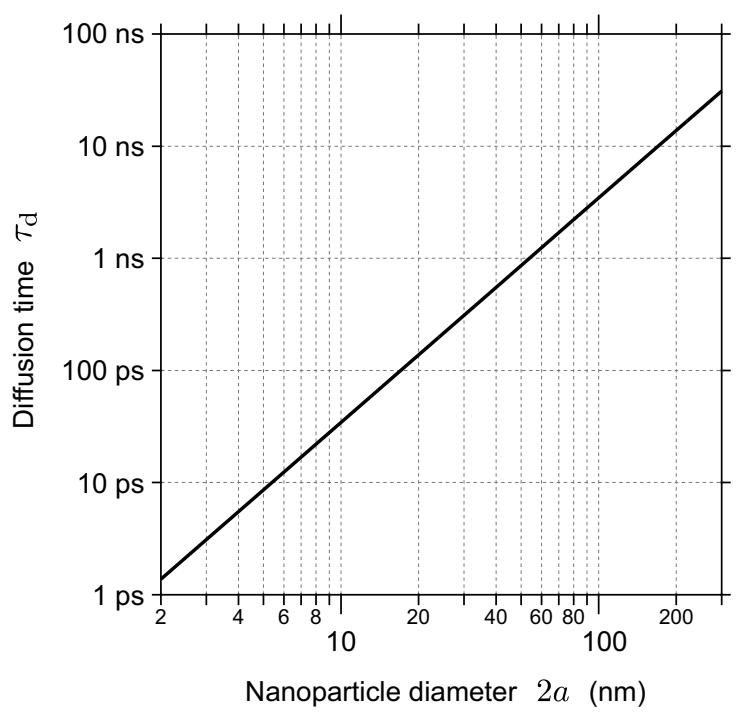

Figure 1: Characteristic time of nanoparticle cooling in water after pulse absorption as a function of the nanoparticle diameter, according to eq 3

From the physical quantities introduced so far, one can derive other useful physical quantities, namely the pulse intensity (or irradiance) $I(t)$ defined as a power per unit area:

$$
I(t)=F q(t)
$$

where $F$ is called the fluence of the pulse (energy per unit area). Note that $\int I(t) \mathrm{d} t=F$. Let us define also the power $P(t)$ transferred from the pulse to the electron gas, which reads

$$
P(t)=\sigma_{\mathrm{abs}} I(t)=\sigma_{\mathrm{abs}} F q(t)
$$

where $\sigma_{\mathrm{abs}}$ is the absorption cross section of the NP. The total energy absorbed by the NP reads thus

$$
E=\int P(t) \mathrm{d} t=\sigma_{\mathrm{abs}} F
$$


A final important physical quantity is the heat power transferred from the electron gas to the atomic lattice (i.e. the phonons) of the NP:

$$
\begin{aligned}
Q(t) & =P \otimes p(t) \\
& =\sigma_{\mathrm{abs}} F q \otimes p(t)
\end{aligned}
$$

where $A \otimes B$ denotes a function that equals the convolution between the functions $A$ and $B$.

One can now discuss the occurrence of different regimes depending on the relative values of the three aforementioned time constants $\tau_{\mathrm{p}}$, $\tau_{\mathrm{ep}}$ and $\tau_{\mathrm{d}}$. For very short pulses such that $\tau_{\mathrm{p}} \ll \tau_{\mathrm{ep}}$, one can consider the function $q$ as a Dirac distribution compared to $p$, which yields $q \otimes p \approx p$. This is typically what happens in the femtosecond regime, since the typical pulse duration of $100 \mathrm{fs}$ is shorter than $\tau_{\mathrm{ep}}=1.7 \mathrm{ps}$. On the contrary, if $\tau_{\mathrm{p}} \gg \tau_{\mathrm{ep}}$, typically with nanosecond pulses, $q \otimes p \approx q$. These approximations make the expression of $Q(t)$ much simpler: it will feature either an exponential decay in the femtosecond range $Q(t)=\sigma_{\mathrm{abs}} F p(t)$, or a Gaussian shape in the nanosecond range $Q(t)=\sigma_{\mathrm{abs}} F q(t)$. The picosecond-pulse range appears therefore as an intermediate regime where $Q(t)$ cannot be approximated by a simple closeform expression, and has to be calculated using eq (8). Note that another approach to determine $Q(t)$ is the so-called two-temperature model (TTM). ${ }^{25,28-30}$ The TTM is a more sophisticated approach, which enables for instance the consideration of non-linearities of the heat capacity of the metal NP as a function of temperature.

\section{Absorption cross section}

The absorption cross section $\sigma_{\mathrm{abs}}$ of a spherical NP can be computed using Mie theory (see appendix A for the theory and supplementary information for the Matlab code). A plot of $\sigma_{\mathrm{abs}}$ for gold is represented in Fig.2. For small radii $a$, typically for a gold NP size $2 a$ below $60 \mathrm{~nm}, \sigma_{\mathrm{abs}}$ is proportional to the NP volume $V=4 \pi a^{3} / 3 .{ }^{9}$ One can thus define a constant $\zeta$ such that

$$
\sigma_{\mathrm{abs}}=\zeta a^{3}
$$


stands for a fitting function of $\sigma_{\mathrm{abs}}(a)$ for small values of $a$. We found $\zeta=0.430 \mathrm{~nm}^{-1}$ for spherical gold NP in water $(n=1.33)$. Such a fit is represented as a dashed line in Fig.2. We will see that such a non-linearity of $\sigma_{\text {abs }}(a)$ with respect to $V$ plays an important role in the physics of bubble generation in plasmonics.

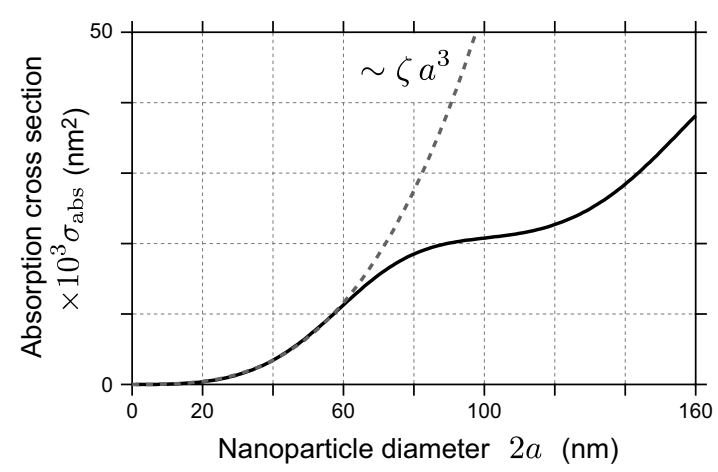

Figure 2: Absorption cross section of a spherical gold nanoparticle in water as a function of its diameter (solid line) for an illumination wavelength of $532 \mathrm{~nm}$. Fitting function for small diameter (dashed line).

\section{Heat diffusion}

Let us consider a spherical gold NP immersed in water. The evolution of the temperature distribution in the system is governed by the heat diffusion equation. ${ }^{31}$ The related set of differential equations reads

$$
\begin{array}{rlrl}
c_{\mathrm{m}} \partial_{t} T-\kappa_{\mathrm{m}} \partial_{\mathbf{r}}^{2} T & =Q(t) / V & & \text { for }|\mathbf{r}|<a \\
c_{\mathrm{w}} \partial_{t} T-\kappa_{\mathrm{W}} \partial_{\mathbf{r}}^{2} T=0 & \text { for }|\mathbf{r}|>a
\end{array}
$$

where $\kappa_{\mathrm{W}}$ is thermal conductivity of water and $c_{\mathrm{W}}$ its volumetric heat capacity. These expressions assume no variations of the properties of the fluid with regard to temperature. To solve the problem, one also has to consider a set of two boundary conditions related to the continuities of the temperature and its gradient at the NP-water interface $|\mathbf{r}|=a$ :

$$
\begin{aligned}
\kappa_{\mathrm{m}} \partial_{\mathbf{r}} T\left(a^{-}\right) & =\kappa_{\mathrm{w}} \partial_{\mathbf{r}} T\left(a^{+}\right) \\
T\left(a^{-}\right) & =T\left(a^{+}\right)
\end{aligned}
$$


The first equation expresses the conservation of thermal flux through the NP interface. The second equation expresses the temperature continuity. Such a continuity may not hold if a surface thermal resistance is present, which is the subject of the next subsection.

\section{Kapitza resistance}

Most interfaces separating different media are endowed with a thermal interface resistance, hindering thermal diffusion. When considering metal nanoparticles in water, a substantial thermal resistance can be favored by the presence of an hydrophobic molecular coating on the NP surface. ${ }^{32-34}$ In this case, the boundary condition related to eq 13 has to be modified. The temperature is no longer continuous across the NP interface. It features a temperature discontinuity that is proportional to the heat flux crossing the interface: ${ }^{31}$

$$
g\left(T\left(a^{+}\right)-T\left(a^{-}\right)\right)=\kappa_{\mathrm{m}} \partial_{\mathbf{r}} T\left(a^{-}\right)=\kappa_{\mathrm{w}} \partial_{\mathbf{r}} T\left(a^{+}\right)
$$

where $g$ is the interface thermal conductivity (a power per unit area and Kelvin). Such a temperature discontinuity makes the temperature in the NP larger than the temperature in the liquid.

In the steady state, i.e. under continuous wave illumination, the presence of a Kapitza resistance leads to a higher NP temperature by a factor of $\left(1+\kappa_{\mathrm{w}} / g a\right) \cdot{ }^{24,35}$ The particular value of $g$ has, however, no effect on the temperature profile in the surrounding medium. This counter-intuitive statement is illustrated in Fig.3.

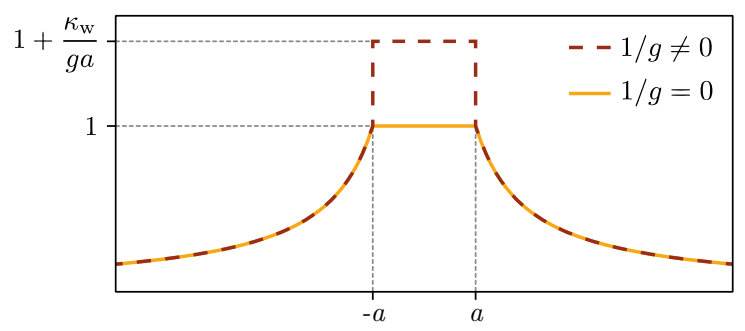

Figure 3: Steady-state normalized temperature profile of a spherical metal nanoparticle of radius $a$ in water. Two cases are represented, the case of zero interface resistivity (solid line) and the presence of an interface resistivity (dashed line).

In transient regime, however, this rule no longer holds and the temperature evolution outside the nanoparticle may depend on the Kapitza resistivity. In particular, a finite thermal conductivity tends to 
slow down heat release to the surroundings. ${ }^{31}$ The associated time scale for heat release in the presence of a finite value of $g$ reads:

$$
\tau_{g}=\frac{a c_{\mathrm{m}}}{3 g}
$$

This time scale has to be compared with $\tau_{\mathrm{d}}$ (see eq (3)). If $\tau_{g} \gg \tau_{\mathrm{d}}$, the heat diffusion dynamics is governed by $\tau_{g}$.

\section{Spinodal temperature and bubble formation}

It has been established that bubble formation occurs around localized nano-absorbers when the temperature of the liquid reaches its spinodal temperature $T_{\mathrm{s}},{ }^{36-38}$ a temperature usually much higher than its boiling point $T_{\mathrm{b}}$. For water at ambient pressure, while $T_{\mathrm{b}}=100^{\circ} \mathrm{C}, T_{\mathrm{s}}=277^{\circ} \mathrm{C}(550 \mathrm{~K}) .{ }^{39}$ Between $T_{\mathrm{b}}$ and $T_{\mathrm{s}}$, the liquid is in a metastable state and one says that superheating occurs. The occurrence of superheating is not restricted to small scale systems. It can occur at the macroscopic scale as well, provided there is no nucleation point (such as dust or scratches). ${ }^{3}$ Sometimes in the literature, the spinodal temperature is not considered as the threshold temperature for bubble formation in plasmonics. Instead, the critical temperature is mentioned. We believe this is not correct. In some other articles, one can read that bubble formation was observed to occur at " $85 \%$ of the critical temperature". But this is precisely where the spinodal temperature stands. We think that no reference should be made to the critical temperature in the context of bubble formation as it has nothing to do with the underlying physics.

\section{Ideal temperature increase $\delta T_{\mathrm{NP}}^{0}$}

A simple close-form estimation of the NP temperature increase can be obtained if we assume no heat release to the surroundings during pulse absorption (denoted by approximation $\mathscr{A}_{1}$ ). Under this assumption, the temperature increase $\delta T=T-T_{0}$ within the NP after pulse absorption follows the relation

$$
E=V c_{\mathrm{m}}\left(T-T_{0}\right)
$$


where $T_{0}$ is the ambient temperature. Due to energy conservation and using eq (6), one obtains a simple expression of the NP temperature increase:

$$
\delta T_{\mathrm{NP}}^{0}=\frac{\sigma_{\mathrm{abs}} F}{V c_{\mathrm{m}}}
$$

One can make a second assumption to further simplify the expression of $\delta T_{\mathrm{NP}}^{0}$. For small NPs (denoted by approximation $\left.\mathscr{A}_{2}\right), \sigma_{\text {abs }}$ is proportional to the NP volume according to eq (9), which yields

$$
\delta T_{\mathrm{NP}}^{0}=\frac{3 \zeta F}{4 \pi c_{\mathrm{m}}}
$$

This is what we call the ideal temperature increase, as it gives the upper limit of the temperature increase than can be observed in the surrounding liquid for a given fluence $F$. Interestingly, $\delta T_{\mathrm{NP}}^{0}$ is not dependent on the NP volume as $a^{3}$ cancels out with the NP volume $V$. This means that if $\mathscr{A}_{1}$ and $\mathscr{A}_{2}$ were both valid for any NP radius, the temperature increase of a nanoparticle after a pulse absorption should be independent of the NP size.

Note that this result only applies at a given fluence. For a given irradiance (power per unit area), i.e. in the case of continuous wave illumination, under the assumption $\mathscr{A}_{2}$ the temperature increase quadratically depends on the nanoparticle diameter.

\section{Ideal fluence threshold $F_{\mathrm{s}}^{0}$}

A first simple close-form estimation of the fluence threshold for bubble formation can be obtained if we assume no heat release to the surroundings during pulse absorption (approximation $\mathscr{A}_{1}$ ). Using eq (17), one obtains

$$
F_{\mathrm{s}}=\frac{4 \pi a^{3} c_{\mathrm{m}}}{3 \sigma_{\mathrm{abs}}}\left(T_{\mathrm{s}}-T_{0}\right)
$$

One can make a second assumption to further simplify the expression of $F_{\mathrm{s}}$. For small NPs (approximation $\left.\mathscr{A}_{2}\right), \sigma_{\text {abs }}$ is proportional to the NP volume according to eq (9), which yields

$$
F_{\mathrm{s}}^{0}=\frac{4 \pi c_{\mathrm{m}}}{3 \zeta}\left(T_{\mathrm{s}}-T_{0}\right)
$$


For gold NPs in water at $20^{\circ} \mathrm{C}$, the ideal fluence threshold equals $F_{\mathrm{s}}^{0} \approx 6.24 \mathrm{~J} \mathrm{~m}^{-2}$. This value stands for a fundamental lower limit related to the association of gold and water. No bubble can be generated around gold nanospheres in water below this fluence. This very simple expression of the fluence threshold $F_{\mathrm{S}}^{0}$, valid under the assumptions of no heat release and small NPs, is interesting as it does not depend on the radius. This is naturally in contradiction with experimental observations - different NP radii are associated to various fluence thresholds - as approximations $\mathscr{A}_{1}$ and $\mathscr{A}_{2}$ are usually not valid, at least rarely at the same time. Approximation $\mathscr{A}_{1}$ leads to an underestimation of the fluence threshold, because any heat release during pulse absorption yields a less pronounced temperature increase, hence the higher required fluence. Assumption $\mathscr{A}_{2}$ also yields an underestimation of $F_{\mathrm{S}}$ as eq (9) overestimates the absorption cross section for most NP diameters.

In order to obtain a proper estimation of the actual fluence threshold to reproduce what has been observed experimentally, both approximations have to be lifted, especially $\mathscr{A}_{1}$, which makes the development of numerical simulations necessary.

\section{Numerical simulations}

Due to the point symmetry of the problem, all the scalar physical quantities, such as the temperature, only depend on the radial coordinate $r=|\mathbf{r}|$ and the problem turns out to be one-dimensional. We solved the problem defined by the set of differential equations and boundary conditions (10), (11), (12) and (14) using a Runge-Kutta-4 (RK4) method. The principle of the algorithm basically consists in discretizing space (as represented in Fig.4) and time. Note that with such a model, we do not make the assumption of NP temperature uniformity. We invite the reader to refer to our previous publication ${ }^{24}$ for more information regarding our RK4 algorithm and to Supplementary Information where we provide the Matlab code.

Different parameters are controlled to ensure the proper convergence of the code. In particular, we plot the total energy of the system as a function of time, calculated from the temperature profile:

$$
e(t)=\int_{0}^{a} 4 \pi r^{2} c_{\mathrm{m}} T(r, t) \mathrm{d} r+\int_{a}^{\infty} 4 \pi r^{2} c_{\mathrm{w}} T(r, t) \mathrm{d} r
$$




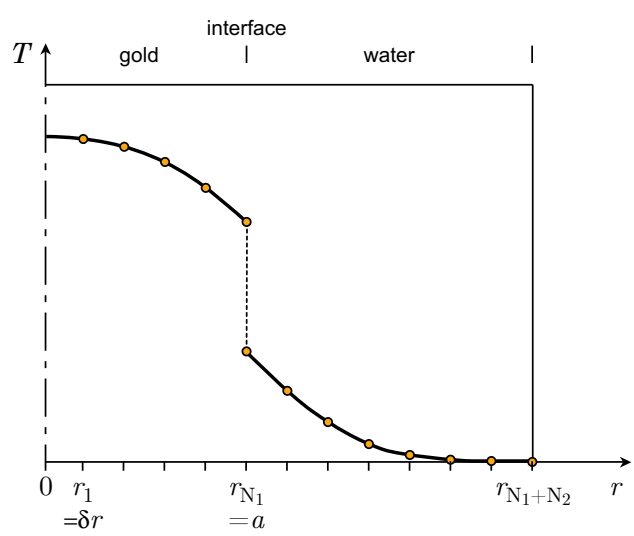

Figure 4: Schematic representing the spatial discretization of the system. The NP-liquid interface is located at $r=a$ where a temperature discontinuity due to a finite Kapitza conductivity is represented.

In a converging simulation, this energy must equal the total energy transferred to the system $\sigma_{\mathrm{abs}} F$ (as defined by eq 6) once the pulse energy transfer is over, and should remain constant afterward.

Some typical numerical results are presented in Fig.5. We have plotted successive temperature profiles $T\left(r ; t_{i}\right)$ for different times $t_{i}$ (Fig.5a). We have also represented the temporal evolution of the energy contained in the system $e(t)$ normalized (divided) by $\sigma_{\mathrm{abs}} F$ (Fig.5b). As expected, the energy $e(t)$ remains constant as soon as the input power $Q(t)$ vanishes. The normalization by $\sigma_{\mathrm{abs}} F$ implies that this plot has to tend to unity, which was ensured in each numerical simulation within an error bar of $\pm 3 \%$. In Fig.5c, the evolution of the temperature inside $\left(T\left(a^{-}, t\right)\right)$ and outside $\left(T\left(a^{+}, t\right)\right)$ the NP are represented. The fact that they do not coincide stems from the finite value of the thermal conductivity $g$, which was set to 150 MW $\mathrm{m}^{-2} \mathrm{~K}^{-1}$ in this particular simulation, a common value reported in the literature. This simulation concerns a particular case where the fluence $F$ equals the fluence threshold $F_{\mathrm{s}}$. Indeed, the maximum temperature achieved over time at the vicinity of the NP reaches the spinodal temperature of water $277^{\circ} \mathrm{C}$.

\section{Results}

In the context of this work, we restricted our simulations to two extreme cases in terms of pulse duration. The first case, termed $f$ s-pulsed regime, is related to the situation where the pulse duration is much shorter than $\tau_{\mathrm{ep}}$. In this case, the exact pulse duration does not matter. The pulse duration is not even a parameter of our numerical simulation, because the heat delivery to the electron gas occurs 
1

2

3

4

5

6

7

8

9
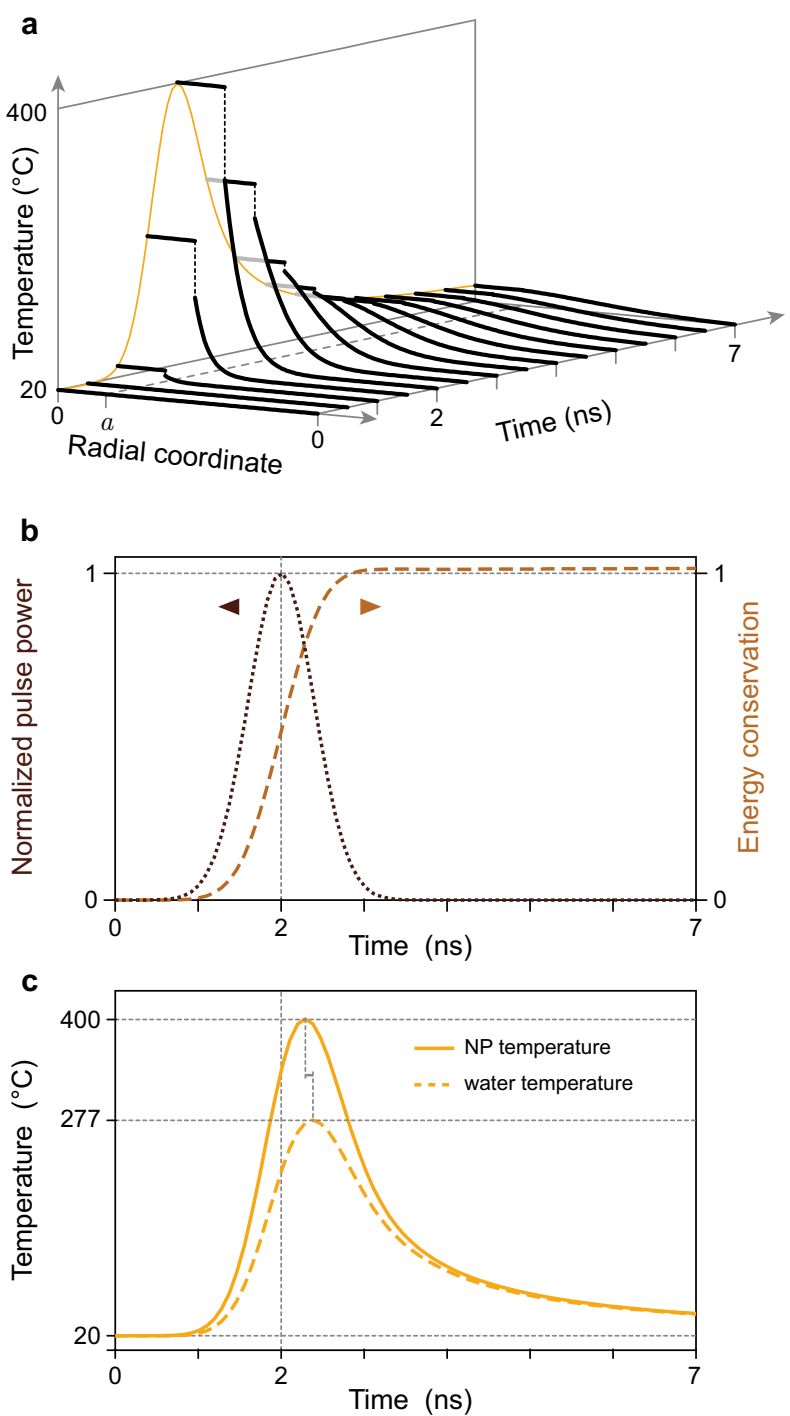

Figure 5: Typical results of an RK4 numerical simulation of the temperature evolution of a spherical nanoparticle in water illuminated by a pulse of light. $\tau_{\mathrm{p}}=1 \mathrm{~ns}, t_{0}=2 \mathrm{~ns}, F=38 \mathrm{~J} \mathrm{~m}^{-2}, a=20 \mathrm{~nm}, g=150$ MW m ${ }^{-2} \mathrm{~K}^{-1}$. Total duration of the simulation: $458 \mathrm{~s}$. (a) Temperature distribution at successive times (every $0.5 \mathrm{~ns}$ ). (b) Time trace of the normalized heating power $Q(t) / \max (Q)$ (dotted line). Time trace of the energy contained in the system (atomic lattice of the nanoparticle, and surrounding water) normalized by the total pulse energy $\sigma_{\mathrm{abs}} F$ (dashed line). (c) Evolution of the temperature on the nanoparticle surface (solid line) and of the water at the vicinity of the nanoparticle (dashed line).

over a time scale $\tau_{\mathrm{ep}}$ according to the profile $p(t)$. Experimentally, it typically corresponds to the use of Ti:Sapphire lasers, which feature a pulse duration of around $200 \mathrm{fs}$. The second case, termed $n s$-pulse regime, is related to a pulse duration of $\tau_{\mathrm{p}}=1 \mathrm{~ns}$. In this case, the heat delivery to the electronic gas follows the profile $q(t)$, over a time scale $\tau_{\mathrm{p}}$.

In these two regimes, and for a series of different NP diameters $2 a$, we calculated the spatio-temporal evolution of the temperature anywhere in the system (inside the NP and in the surrounding fluid). The 

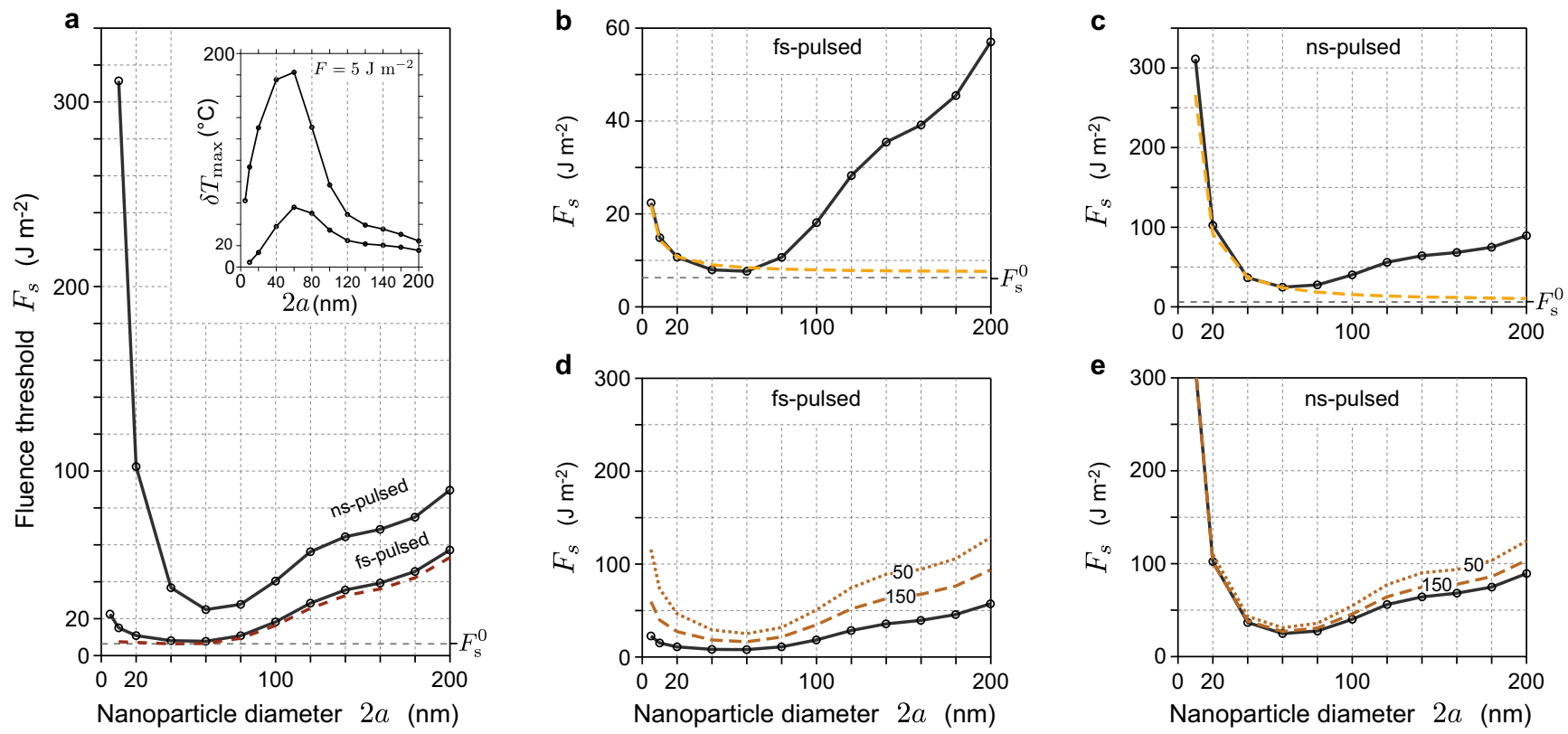

Figure 6: (a) Fluence threshold calculated for a 1-ns pulse and for a femtosecond pulse (solid lines), as a function of the NP diameter. The dashed line represents the fluence threshold in the no-diffusion regime. The inset represents the maximum temperature achieved in the surrounding water as a function of the NP diameter, for a given fluence of $F=5 \mathrm{~J} \mathrm{~m}^{-2}$. (b) Fluence threshold in the femtosecond regime (solid line) compared with the fluence threshold calculated assuming a linear relation between the absorption cross section and the NP volume (dashed line). (c) Same as (b) in the case of a 1-ns pulse. (d) Fluence threshold in the femtosecond regime (solid line) compared with the fluence threshold related to a Kapitza conductivity of $50 \mathrm{MW} \mathrm{m}^{-2} \mathrm{~K}^{-1}$ (dotted line) and $150 \mathrm{MW} \mathrm{m}^{-2} \mathrm{~K}^{-1}$ (dashed line). (e) Same as (d) in the case of a 1-ns pulse. The data related to all these graphs is provided in Supplementary Materials as an Excel file. 
a

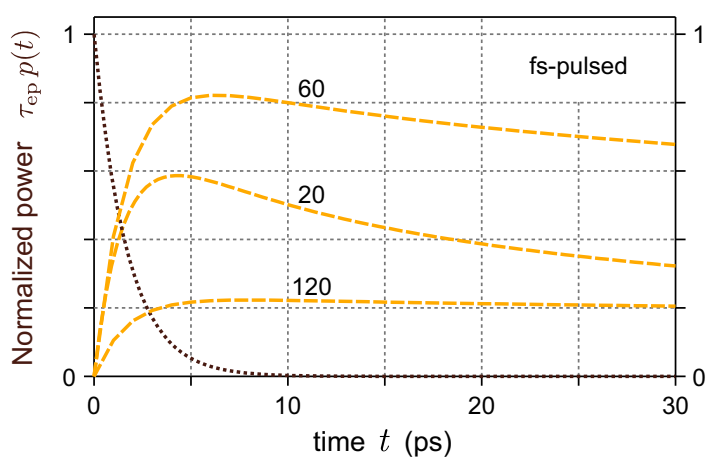

b

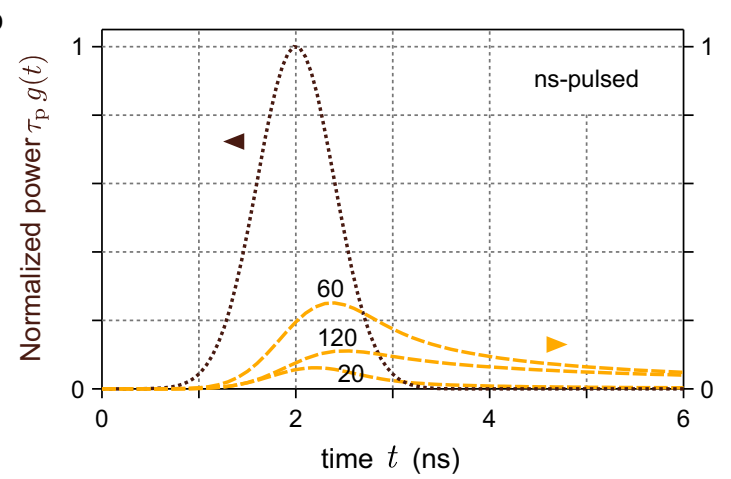

Figure 7: (a) (dotted line) Normalized power $p(t)$ transferred to the nanoparticle as a function of time (see eq (2)), corresponding to the case of a femtosecond pulse. (dashed lines) Corresponding evolutions of the temperature of the nanoparticle normalized by the ideal temperature increase $T_{\mathrm{NP}}^{0}$, for three different nanoparticles diameters: 20, 60 and $120 \mathrm{~nm}$.(b) (dotted line) Normalized power $q(t)$ transferred to the nanoparticle as a function of time (see eq (1)), corresponding to the case of a nanosecond pulse of duration $\tau_{\mathrm{p}}=1 \mathrm{~ns}$. (dashed lines) Corresponding evolutions of the temperature of the nanoparticle normalized by the ideal temperature increase $T_{\mathrm{NP}}^{0}$, for three different nanoparticles diameters: 20, 60 and $120 \mathrm{~nm}$. 
inset of Fig.6a displays the maximum water temperature $\delta T_{\max }$ achieved over time as a function of the NP diameter (solid lines), for a pulse fluence $F_{0}=5 \mathrm{~J} \mathrm{~m}^{-2}$. From these plots, the fluence threshold was retrieved using

$$
F_{\mathrm{s}}=F_{0} \frac{T_{\mathrm{s}}-T_{0}}{\delta T_{\max }}
$$

This relation assumes a linearity of the temperature with respect to the pulse fluence. The corresponding plots of $F_{\mathrm{S}}$ are represented in Fig.6a. These profiles constitute the central result of our work. A bathtub shape is evidenced, both for the nanosecond and femtosecond regimes, in agreement with previous experimental and theoretical works. ${ }^{1,19,21}$ In particular, a minimum is found for a NP diameter of around $60 \mathrm{~nm}$, no matter the pulse duration. We shall now deeper investigate the origin of this bathtub shape.

Let us first focus on the origin of the abrupt fluence threshold increase observed at small NP diameters. In Fig.6a, the dashed line represents the calculated fluence threshold using eq (19), i.e. under the assumption of no-diffusion in the surrounding medium (approximation $\mathscr{A}_{1}$ ). This line shape almost matches the fs-pulsed regime, except for small NP sizes. The fact that the line shapes do not coincide for small NP sizes means that approximation $\mathscr{A}_{1}$ is not valid for small NP radii. Consequently, the abrupt fluence threshold increase observed experimentally for small NP diameters results from heat diffusion in the surrounding medium. Conversely, for NP larger than $\sim 40 \mathrm{~nm}$, heat diffusion in the surrounding does not play any role in the underlying physics of fluence threshold. It is all as if no diffusion were occurring in the surroundings during NP heating, and the proper estimation of the fluence threshold can be achieved without conducting numerical simulations, just using the expression of the ideal temperature increase (19). Only $\sigma_{\mathrm{abs}}$ has to be computed, eventually using Mie theory (see Appendix).

Regarding the ns-pulsed regime, the divergence at small radii is also due to a significant heat release to the surroundings during pulse duration. The effect is however more pronounced compared to the fs-pulsed regime because the heating of the NP occurs over the nanosecond time scale, not the picosecond time scale. Consequently, much more energy escapes during the pulse. Note that the deviation from the ideal fluence threshold (dashed line) in the ns-pulse regime remains even for large NPs. This is because even for NPs as large as $200 \mathrm{~nm}$, the time scale of heat diffusion $\tau_{\mathrm{d}} \approx 10 \mathrm{~ns}$ remains on the order of the pulse duration $\tau_{\mathrm{p}}=1 \mathrm{~ns}$. Consequently, under ns-pulsed illumination, the ideal fluence threshold can never be 
achieved, no matter the pulse duration or the NP size.

Another intriguing aspect is a systematic increase of the fluence threshold $F_{\mathrm{s}}$ for large NP radii. To understand this observation, we have plotted a calculation of $F_{\mathrm{s}}$ under the assumption of linear dependence of the absorption cross section with respect to the volume of the NP (approximation $\mathscr{A}_{2}$ ) in Figs.6b,c. As expected, a mismatch is observed for NPs larger than $60 \mathrm{~nm}$, because $\sigma_{\mathrm{abs}}$ is no longer proportional to $V$ from this value, according to Fig.2. But more interestingly, this approximation would lead to a constant fluence threshold for large NP radii. The fluence threshold increase observed for large NPs therefore results from the damping of the absorption cross section above $2 a=60 \mathrm{~nm}$.

In order to further illustrate the above-mentioned conclusions, time traces of pulse power and water temperature are plotted in Fig. 7 for $2 a=20,60$ and $120 \mathrm{~nm}$ (in the case of no interface resistivity). Temperatures have been normalized by the ideal temperature increase $T_{\mathrm{NP}}^{0}$ so that unity is the maximum value that can be achieved. In the fs-pulsed regime (Fig.7a), for $2 a=120 \mathrm{~nm}$, one can see than even though the heat release to the surroundings is very slow, the normalized temperature increase remains far from unity, illustrating the effect of the non-linearity of the absorption cross section. In the case $2 a=20 \mathrm{~nm}$, the maximum temperature increase remains far from unity as well, but for a different reason. The temperature increase is affected by fast heat diffusion in the surroundings, because small NPs are associated to fast heat release in the surroundings (according to eq (3)). The optimal case of $2 a=60 \mathrm{~nm}$ has been represented. In the ns-pulsed regime (Fig.7b), any normalized temperature line shape remains far from unity as diffusion plays a dominant role. No slow temperature decrease is observed for any NP size.

Another parameter that we shall investigate is the NP-liquid interface resistivity $1 / g$. Results are illustrated by Figs.6d,e, where dashed lines refer to numerical simulations including thermal conductivities of $g=150 \mathrm{MW} \mathrm{m}{ }^{-2} \mathrm{~K}^{-1}$, a typical value reported by the community, ${ }^{32-34}$ and $g=50 \mathrm{MW} \mathrm{m}^{-2} \mathrm{~K}^{-1}$, the lowest reported value, to our knowledge. ${ }^{34}$ The general conclusion is that a finite thermal conductivity has strong effect in fs-pulsed illumination, but has weak effect on the ns-pulsed illumination. This is a general rule of thumb, which can be explained by comparing the different time scales coming into play. According to eq (15), $\tau_{\mathrm{g}}$ ranges from $40 \mathrm{ps}$ to $3 \mathrm{~ns}$ for the range of NP diameters investigated herein. Under pulsed illumination, the heat delivery occurs over a time scale that equals $\tau_{\mathrm{ep}}=1.7 \mathrm{ps}$, which is much smaller than 

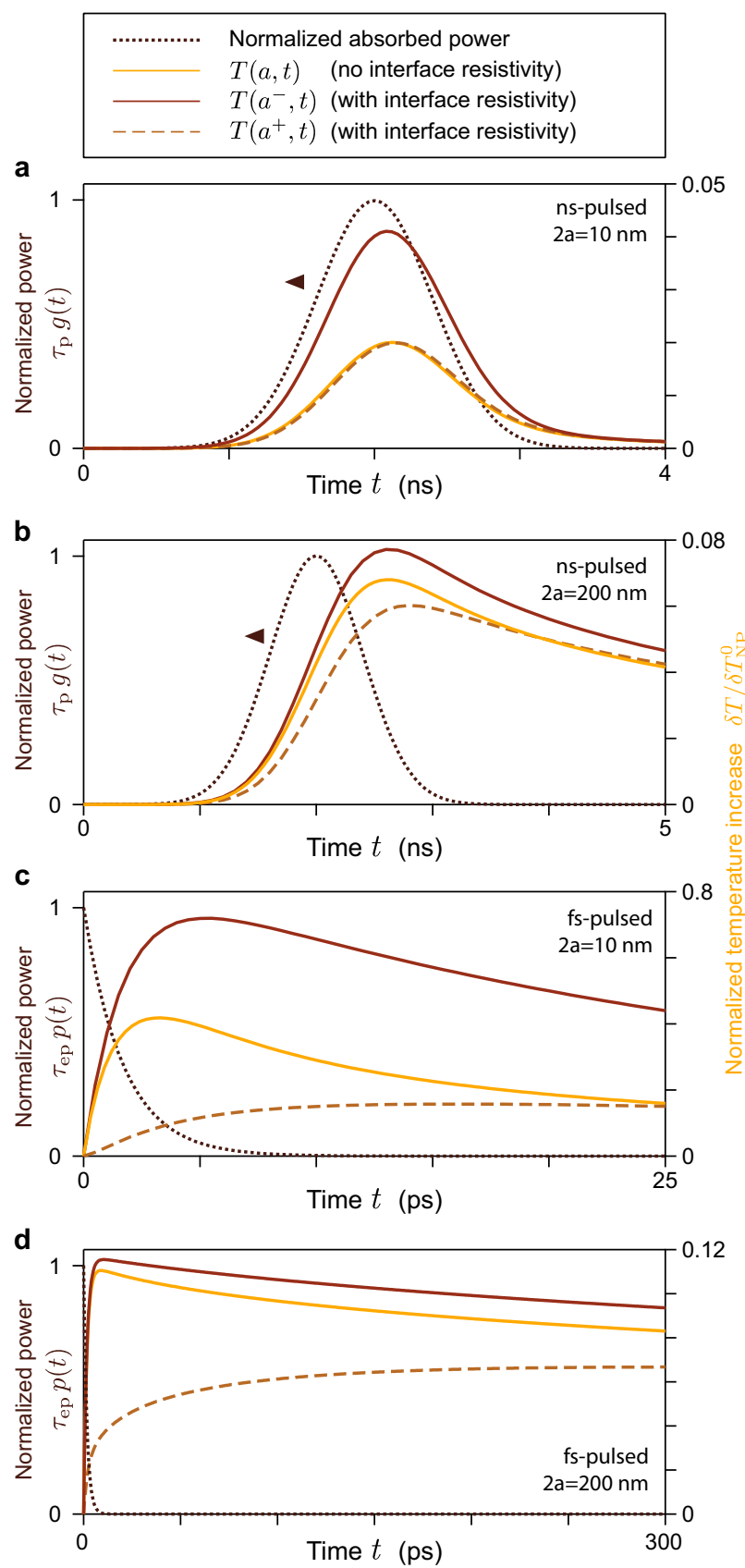

Figure 8: Evolutions of the normalized power transferred to the nanoparticle as a function of time, and the associated temperature profiles at the nanoparticle interface. (a) Case of a 10-nm NP and a 1-ns pulse. (b) Case of a 200-nm NP and a 1-ns pulse. (c) Case of a 10-nm NP and a fs-pulse. (d) Case of a 200-nm NP and fs-pulse. 
$\tau_{\mathrm{g}}$ for any NP size. Consequently, no matter the NP size, the heat release from the NP to the surrounding liquid is always delayed due to the finite interface thermal conductivity, hence the reduced temperature increase in the surrounding medium. The delay is illustrated in Figs.8c,d, where the evolutions of the temperatures inside and outside a NP have been plotted. One can see that the temperature outside the NP evolves much more slowly than the temperature inside the NP. Under ns-pulsed illumination, such a delay is not supposed to occur as the time scale of NP heating $\left(\tau_{\mathrm{p}}=1 \mathrm{~ns}\right)$, which is much slower than $\tau_{\mathrm{g}}$. Consequently, the NP surface resistivity does not contribute to further slow down the heat exchange between the NP and the surrounding medium. In Fig.8a, corresponding to a small NP in the ns-pulsed regime, one can even observe that the temperature outside the NP does not depend on the presence of a surface resistance (dashed and orange solid lines are superimposed). This is a characteristic feature of a steady state (see previous section on Kapitza resistance and the associated Fig.3). Consequently, one can consider that the temperature dynamics of small NPs under the ns-pulsed regime can be described by a quasistatic evolution. For large NPs (Fig.8b), a small deviation can be observed in Fig.6e. This is when $\tau_{\mathrm{g}}$ becomes on the order of magnitude of $\tau_{\mathrm{p}}$, which creates a delay just like under fs-pulsed illumination in Fig.8d.

The last parameter that we shall discuss is the wavelength. So far, all the calculations have been carried out using an illumination wavelength of $532 \mathrm{~nm}$, matching both the resonance of small gold spheres and a common laser wavelength. However, other wavelengths are sometimes used experimentally, such as $400 \mathrm{~nm}^{19}$ or $355 \mathrm{~nm} .{ }^{1,19,21}$ One may wonder whether the main trends we observed for a wavelength of $532 \mathrm{~nm}$ are still valid at other wavelengths. The answer is basically yes, as illustrated by Fig.9. We chose to represent a wavelength of $355 \mathrm{~nm}$ (400 nm gives very similar results). At this wavelength, the plasmonic resonance efficiency is naturally reduced (weaker absorption cross section as represented in Fig.9a), which tends to increase the required laser fluence to generate a bubble. According to these results, the minimum fluence observed around $2 a=60 \mathrm{~nm}$ in the case of a wavelength of $532 \mathrm{~nm}$ still holds at this other wavelength. In other words, this bathtub shape is not a particularity of the illumination at $532 \mathrm{~nm}$, but rather a general rule. 

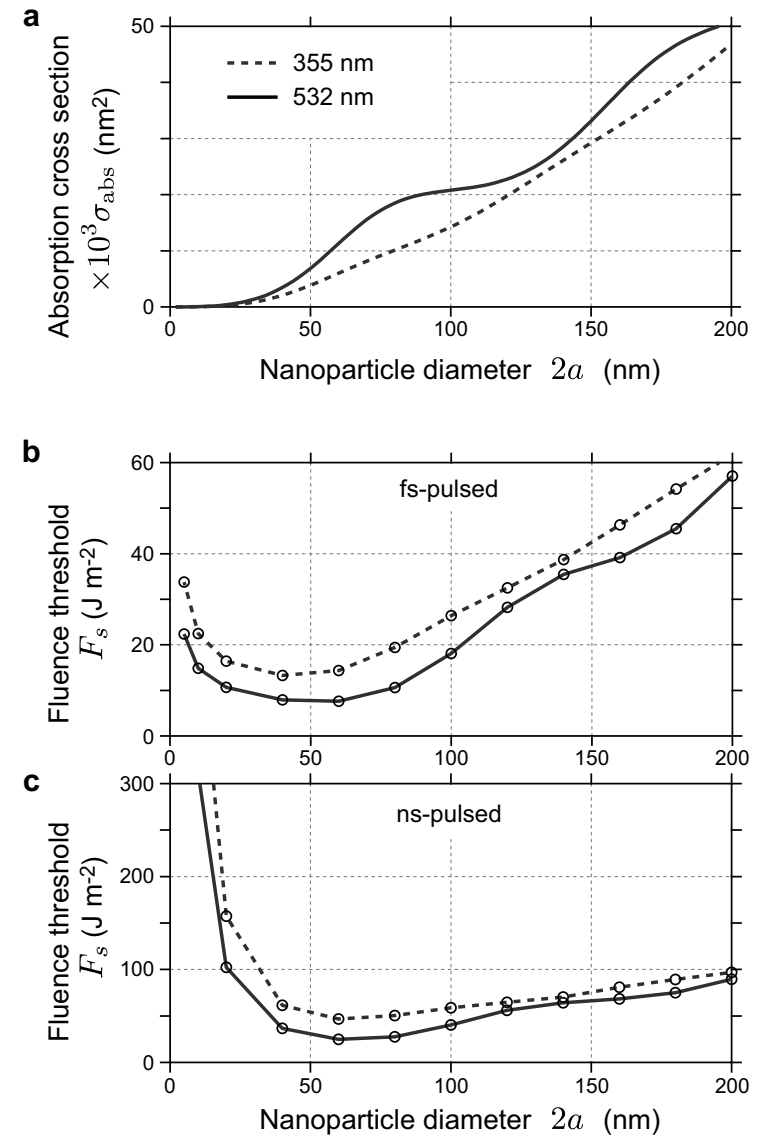

Figure 9: (a) Absorption cross sections of spherical gold particles in water as a function of their diameter and for two different wavelengths: $355 \mathrm{~nm}$ (dashed line) and $532 \mathrm{~nm}$ (solid line). (b) Fluence thresholds as a function of the nanoparticle diameter for both wavelengths, in the case of the fs-pulsed regime. (c) Fluence thresholds as a function of the nanoparticle diameter for both wavelengths, in the case of the ns-pulsed regime. 


\section{Discussion}

This last section is mainly intended to (i) discuss the approximation we have made in our formalism to simplify the discussions, and (ii) compare our results to previous reported studies in the literature.

Our formalism is linear in any of its aspects. In particular, we assume no temperature dependence of the thermal conductivity, heat capacity and Kapitza resistance. The purpose of this article is to explain the main trends governing the magnitude of the fluence threshold (in particular this bathtub shape). As these main trends are not governed by any non-linearity, it becomes natural to discard these effects.

We focused on spherical geometries, and not on more complicated morphologies, for several reasons. (i) Spherical NPs are easier to model numerically. (ii) Many experimental investigations are carried out using spherical NPs anyway. (iii) All the underlying physics can be described with a simple spherical model. (iv) Non-spherical shapes, like rods, may involve other physical processes for bubble formation that have nothing to do with thermal effects, like the formation of a plasma as described in particular by the Meunier's group in the femtosecond regime. ${ }^{40,41}$

All the calculated fluence thresholds of our work are slightly underestimated compared to experimental measurements. There are different possible reasons for that. (i) We consider the specific wavelength of $532 \mathrm{~nm}$, close to the optimal absorption wavelength for spherical NPs. All the reported experiments do not necessarily use a wavelength that matches the plasmon resonance of the NPs. (ii) We considered that bubble formation occurs when the fluid temperature reaches $T_{\mathrm{s}}$ at $r=a$. Although this assumption may seem natural, it is known to yield underestimated values. ${ }^{21}$ In order to better match experimental values, it was proposed to use a different criteria for bubble generation, that is a temperature increase up to $T_{\mathrm{S}}$ over a given volume around the NP corresponding to a thickness $e$ of typically $e=10 \mathrm{~nm} .{ }^{21}$ We could have easily used this criteria, but this would have added another parameter (the water layer thickness $e$ ), which would have not qualitatively changed our conclusions. (iii) Right at the fluence threshold we predict, a bubble could appear experimentally, but be too small, scatter too less or collapse too fast to be detectable. The fact that different groups have reported different fluence thresholds for the same experimental conditions is a sign that the determination of bubble formation may depend on the sensitivity of the detection. This problem may be even more significant when conducting measurements on single NPs instead ensemble 
measurements, due to a reduced signal to noise ratio. (iv) A last effect that could be at the origin of a mismatch between experiment and numerical simulations is the dispersion in a colloidal NP batch, in shape and sphericity, as noted by Siems et al., ${ }^{19}$ especially for NP diameters larger than 70-80 nm.

We shall now review what has been discussed in the literature regarding fluence threshold determination in plasmonics and compare it with our results.

In 2010, Lukianova-Helb et al. ${ }^{1}$ experimentally investigated the fluence threshold dependence as a function of the NP diameter in the ns-pulsed regime $\left(\tau_{\mathrm{p}}=0.5 \mathrm{~ns}\right)$ for a set various NP diameters, namely 10, 30, 60, 80 and $250 \mathrm{~nm}$. They found a minimum fluence threshold for a NP diameter of $80 \mathrm{~nm}$. Like in several other reported studies, they explained the strong fluence threshold increase for small NPs with surface tension considerations: According to them, overcoming the Laplace pressure would require higher energies for small NPs. This is not in agreement with the interpretation we provide in this article, which rather evidences a faster energy release to the surroundings during the pulse duration. We do not think that a Laplace pressure can be at the origin of the absence of nucleation because any consideration of a Laplace pressure mechanism already supposes the presence of a bubble, which is paradoxical. Thus, the absence of nucleation, i.e. the occurrence of superheating, can be simply explained only by considering a real gas with molecular interactions, for instance described by a van der Waals equation of state. ${ }^{38,39}$ With such an approach, one easily realizes that the absence of bubble formation comes from long-range attraction of the fluid's molecules, which acts as a restoring force when the fluid tends to locally expand due thermal fluctuations. This restoring force only vanishes at the spinodal temperature. We believe that invoking Laplace pressure or surface tension for this process is misleading as there is no creation of liquid-gas interface, strictly speaking.

In 2011, Siems et al. ${ }^{19}$ investigated the fs-pulsed and ns-pulsed regimes. Supported by numerical simulations, the fs-pulsed regime was investigated experimentally by X-ray scattering and the ns-pulsed regime was investigated experimentally by optical means. Their numerical approach, based on a Laplace transform formalism and the use of analytical expressions, is different from ours and does not lead to the same conclusions. In particular, in the nanosecond regime, they predict higher fluence thresholds than we do. And in the femtosecond regime, they do not predict an increase of the fluence threshold for nanoparticles larger than $60 \mathrm{~nm}$. Their theoretical fluence threshold profile remains monotonic up to 
$2 a=100 \mathrm{~nm}$. Regarding their experimental measurements, the nanosecond pulse regime was investigated only up to a NP radius of $60 \mathrm{~nm}$. Regarding the fs-pulsed regime, NP diameters between 50 and $90 \mathrm{~nm}$ have not been investigated and the measurements are rather dispersed. The absence of fluence threshold minimum at $60 \mathrm{~nm}$ cannot be clearly evidenced by their experimental data.

In 2014, Katayama et al. ${ }^{21}$ reported on an experimental study of bubble generation using a ps-pulsed illumination of 20-150 nm gold NPs at $\lambda=355 \mathrm{~nm}$. They conducted a rich discussion on the underlying physics of bubble formation, and in particular on the fluence threshold. They do observe a minimum fluence threshold at a NP diameter of $60 \mathrm{~nm}$, in agreement with our numerical predictions (see Fig.6 and Fig.9c). The occurrence of a non-monotonic shape of $F_{\mathrm{s}}(a)$ with a minimum at $60 \mathrm{~nm}$ is also supported by the results of Cavicchi et al. ${ }^{42}$ who investigated nanoparticle reshaping dependence on laser fluence. In the article, Katayama et al. ${ }^{21}$ proposed that this non-monotonic dependence of $F_{\mathrm{S}}(a)$ and this dip at 60 $\mathrm{nm}$ can ascribed to the variations of the quantity $\left(\sigma_{\mathrm{abs}} / V\right) / g_{\mathrm{c}}$, where $g_{\mathrm{c}}$ is the critical thermal interface conductance. ${ }^{43}$ This interpretation in not in agreement with the interpretation we propose. According to our results, a thermal resistivity at the NP interface is not supposed to play a role in this non-monotonic shape. Indeed, in Fig.6a, a dip at $60 \mathrm{~nm}$ is observed even if no interface resistivity is involved. We shall now further discuss the use of $g_{\mathrm{c}}$ in the context of bubble nucleation in plasmonics, which is, we believe, much more complicated than what was described in the literature. $g_{\mathrm{c}}$ is defined by equating the diffusion time scale in water $a c_{\mathrm{w}} / 3 \kappa_{\mathrm{w}}$ and $\tau_{\mathrm{g}}$ defined by eq (15). It yields

$$
g_{\mathrm{c}}=\frac{3 c_{\mathrm{w}} \kappa_{\mathrm{w}}}{c_{\mathrm{m}} a}
$$

Consequently, $g_{\mathrm{c}}$ is expected to tell when the surface resistivity plays a role or not. According to this expression, a given surface resistivity is supposed to play a role for small radii and less for large radii. This trend is just the opposite of what we observed in our numerical results (see Fig.6e). On the contrary, the presence of a surface resistivity is more significant for large NPs and nonexistent for small NPs. The origin of this paradox is intricate. Actually, in the ns-pulse regime, when the NP is too small, the heat release to the surrounding is so fast that it forces the system to be in the quasistatic regime, as already explained in Fig.8a (the temperature profile scaling as $1 / \mathrm{r}$ is achieved any time in the surrounding medium). The direct consequence is no effect of the Kapitza resistivity in the surrounding medium, because this is what is 
supposed to happen in the steady state, as explained in the introductory section of this article (see Fig.3a). In other words, the very fast release of heat in the surrounding medium kills the effect of the Kapitza resistivity in the surrounding medium as it forces a quasistatic regime. However, the effect of the Kapitza resistivity inside the NP is strong (see Fig.3). When the NP becomes larger, the heat release becomes slower according to eq (3), the system deviates from the quasistatic state, and the interface resistivity is now playing a role in the surrounding medium as well. The other drawback of $g_{\mathrm{c}}$ is that it does not involve the pulse duration. We have shown yet that the presence of a Kapitza resistivity does not lead to the same trends under femtosecond and nanosecond regimes (see the comparison between Figs.6d and e). As a conclusion, we do not believe that $g_{\mathrm{c}}$ is a proper parameter to be used in the context of bubble nucleation in plasmonics.

\section{Conclusion}

In conclusion, we have numerically investigated the physics of bubble nucleation around gold nanospheres in order to account for previous reported observations. In particular, we explained the bathtub shape of the fluence threshold dependence as a function of the nanoparticle size. Here is a summary of the main results of this work regarding the underlying physics of bubble generation around gold nanospheres in water. (i) The dependence of the fluence threshold with respect to the nanoparticle diameter features a bathtub profile, with a minimum fluence threshold (maximum bubble generation efficiency) at around 60 $\mathrm{nm}$ in diameter. (ii) The fluence threshold increase for small NPs stems from fast energy release to the surroundings. (iii) The fluence threshold increase for large NPs results from the non-linearity of the absorption cross section with respect to the NP volume. (iv) Under fs-pulsed illumination, and under the assumption of no interface resistivity, the maximum temperature increase can be simply calculated using eq (17), without conducting numerical simulations, except for NP diameters smaller than $40 \mathrm{~nm}$. (v) A NP interface thermal resistivity has no effect under ns-pulsed illumination, except eventually for large NPs and large values of the interface resistivity. (vi) A NP interface thermal resistivity has a strong effect in the fs-pulsed regime, irrespectively of the nanoparticle diameter.

This work is expected to clarify the physics of bubble nucleation in plasmonics under pulsed illumination. Indeed, most of our conclusions are in good agreement with previous reported experimental results, 
but not with all the reported interpretations. This work may help investigate new routes to the design of more efficient bubble nanosources.

\section{Acknowledgments}

The authors wish to acknowledge the Institut Carnot STAR, which financially supported this work through the MUSEM 2015-IC-STAR project.

\section{Appendix A: Mie theory}

The reference document on Mie theory is the famous textbook from Bohren and Huffman ${ }^{44}$ but the information is not easy to extract. For this reason, we detail in this Appendix the underlying theory and provide in Supplementary Materials the corresponding Matlab code we used in this article to compute the absorption cross section of spherical gold nanoparticles.

Consider a spherical particle of radius $r_{0}$, complex electric permittivity $\varepsilon=n^{2}$ embedded in a dielectric medium of permittivity $\varepsilon_{\mathrm{m}}=n_{\mathrm{m}}^{2}$. This particle is illuminated by a plane wave of angular frequency $\omega=$ $2 \pi c / \lambda_{0}=k c / n_{\mathrm{m}}$

Let us define from now on a set of useful dimensionless parameters:

$$
\begin{aligned}
m & =n / n_{\mathrm{m}} \\
v & =k r_{0} \\
w & =m x
\end{aligned}
$$


In these conditions, the extinction, scattering and absorption cross sections are given by the formulae:

$$
\begin{aligned}
\sigma_{\mathrm{ext}} & =\frac{2 \pi}{k^{2}} \sum_{j=1}^{\infty}(2 j+1) \operatorname{Re}\left(a_{j}+b_{j}\right) \\
\sigma_{\mathrm{sca}} & =\frac{2 \pi}{k^{2}} \sum_{j=1}^{\infty}(2 j+1)\left(\left|a_{j}\right|^{2}+\left|b_{j}\right|^{2}\right) \\
\sigma_{\mathrm{abs}} & =\sigma_{\mathrm{ext}}-\sigma_{\mathrm{sca}}
\end{aligned}
$$

where

$$
\begin{aligned}
a_{j} & =\frac{m \psi_{j}(w) \psi_{j}^{\prime}(v)-\psi_{j}(v) \psi_{j}^{\prime}(w)}{m \psi_{j}(w) \xi_{j}^{\prime}(v)-\xi_{j}(v) \psi_{j}^{\prime}(w)} \\
b_{j} & =\frac{\psi_{j}(w) \psi_{j}^{\prime}(v)-m \psi_{j}(v) \psi_{j}^{\prime}(w)}{\psi_{j}(w) \xi_{j}^{\prime}(v)-m \xi_{j}(v) \psi_{j}^{\prime}(w)}
\end{aligned}
$$

In these expressions, $\psi_{j}$ and $\xi_{j}$ are Ricatti-Bessel functions defined as:

$$
\begin{aligned}
\psi_{j}(x) & =\sqrt{\frac{\pi x}{2}} J_{j+\frac{1}{2}}(x) \\
\xi_{j}(x) & =\sqrt{\frac{\pi x}{2}}\left[J_{j+\frac{1}{2}}(x)+i Y_{j+\frac{1}{2}}(x)\right]
\end{aligned}
$$

$J_{v}$ and $Y_{v}$ are the Bessel functions of first and second order respectively. They are standard Matlab functions, named respectively bessel $j$ and bessely. Note that these functions are solutions of the Bessel differential equation:

$$
x^{2} \frac{\mathrm{d}^{2} y}{\mathrm{~d} x^{2}}+2 x \frac{\mathrm{d} y}{\mathrm{~d} x}+\left[x^{2}-v(v+1)\right] y=0
$$

while $\psi_{j}$ and $\xi_{j}$ are solutions of the following differential equation:

$$
x^{2} \frac{\mathrm{d}^{2} y}{\mathrm{~d} x^{2}}+\left[x^{2}-j(j+1)\right] y=0
$$


$\psi_{j}$ and $\xi_{j}$ can be expressed as a sum of sines and cosines. For instance, the first terms read:

$$
\begin{aligned}
& \psi_{0}(x)=\sin (x) \\
& \xi_{0}(x)=\sin (x)-i \cos (x) \\
& \psi_{1}(x)=\sin (x) / x-\cos (x) \\
& \xi_{1}(x)=\sin (x) / x-i(\cos (x) / x+\sin (x))
\end{aligned}
$$

In Eqs. (24) and (25), the sum over $j$ can be restricted to only a few terms, up to $j=N$. Bohren and Huffman $^{44}$ proposed the value $N=v+4 v^{1 / 3}+2$.

In Eqs. (24) and (25), the primes indicate differentiation with respect to the argument in parenthesis. The derivatives can be conveniently expressed as follows:

$$
\begin{aligned}
\psi_{j}^{\prime}(x) & =\psi_{j-1}(x)-\frac{j}{x} \psi_{j}(x) \\
\xi_{j}^{\prime}(x) & =\xi_{j-1}(x)-\frac{j}{x} \xi_{j}(x)
\end{aligned}
$$

\section{References}

1. Lukianova-Hleb, E.; Hu, L., Y. andLatterini; Tarpani, L.; Lee, S.; Drezek, R. A.; Hafner, J. H.; Lapotko, D. O. Plasmonic Nanobubbles as Transient Vapor Nanobubbles Generated around Plasmonic Nanoparticles. ACS Nano 2010, 4, 2109.

2. Kotaidis, V.; Dahmen, C.; von Plessen, G.; Springer, F.; Plech, A. Excitation of Nanoscale Vapor Bubbles at the Surface of Gold Nanoparticles in Water. J. Chem. Phys. 2006, 124, 184702.

3. Baffou, G.; Polleux, J.; Rigneault, H.; Monneret, S. Super-Heating and Micro-Bubble Generation around Plasmonic Nanoparticles under cw Illumination. J. Phys. Chem. C 2014, 118, 4890.

4. Donner, J.; Baffou, G.; McCloskey, D.; Quidant, R. Plasmon-Assisted Optofluidics. ACS Nano 2011, $5,5457-5462$.

5. Ndukaife, J. C.; Mishra, A.; Guler, U.; Nnanna, A. G. A.; Wereley, S. T.; Boltasseva, A. Photother- 
mal Heating Enabled by Plasmonic Nanostructures for Electrokinetic Manipulation and Sorting of Particles. ACS Nano 2014, 8, 9035-9043.

6. Baffou, G.; Quidant, R. Nanoplasmonics for Chemistry. Chem. Soc. Rev. 2014, 43, 3898-3907.

7. Würger, A. Thermal Non-Equilibrium Transport in Colloids. Rep. Prog. Phys. 2010, 73, 126601.

8. Braun, M.; Bregulla, A. P.; K., G.; Mertig, M.; Cichos, F. Single Molecules Trapped by Dynamic Inhomogeneous Temperature. Nano Lett. 2015, 15, 5499-5505.

9. Baffou, G.; Quidant, R. Thermo-plasmonics: Using Metallic Nanostructures as Nano-Sources of Heat. Laser \& Photon. Rev. 2013, 7, 171-187.

10. François, L.; Mostafavi, M.; Belloni, J.; Delaire, J. A. Optical Limitation Induced by Gold Clusters: Mechanism and Efficiency. Phys. Chem. Chem. Phys. 2001, 3, 4965-4971.

11. Pitsillides, C. M.; Joe, E. K.; Anderson, R. R.; Lin, C. P. Selective Cell Targeting with Light Absorbing Microparticles and Nanoparticles. Biophys. J. 2003, 84, 4023.

12. Bendix, P. M.; Reihani, S. N. S.; Oddershede, L. B. Direct Measurements of Heating by Electromagnetically Trapped Gold Nanoparticles on Supported Lipid Bilayers. ACS Nano 2010, 4, 2256.

13. Zhao, C.; Liu, Y.; Zhao, Y.; Fang, N.; Huang, T. J. A Reconfigurable Plasmofluidic Lens. Nature Commun. 2013, 4, 2305.

14. Hou, L.; Yorulmaz, M.; Verhart, N. R.; Orrit, M. Explosive Formation and Dynamics of Vapor Nanobubbles around a Continuously Heated Gold Nanosphere. New. J. Phys. 2015, 17, 013050.

15. Zhao, C.; Xie, Y.; Mao, Z.; Zhao, Y.; Rufo, J.; Yang, S.; Guo, F.; Maic, J. D.; Jun Huang, T. Theory and Experiment on Particle Trapping and Manipulation via Optothermally Generated Bubbles. Lab on a Chip 2014, 14, 384-391.

16. Jaque, D.; Martinez Maestro, L.; del Rosal, B.; Haro-González, P.; Benayas, A.; Plaza, J. L.; Martín Rodriguez, E.; García Solé, J. Nanoparticles for Photothermal Therapies. Nanoscale 2014, 6, 9494-9530. 
17. Kim, C.; Cho, E. C.; Chen, J.; Song, K. H.; Au, L.; Favazza, C.; Zhang, Q.; Cobley, C. M.; Gao, F.; Xia, Y. et al. In Vivo Molecular Photoacoustic Tomography of Melanomas Targeted by Bioconjugated Gold Nanocages. ACS Nano 2010, 4, 4559-4564.

18. Lukianova-Hleb, E.; Lapotko, D. O. Experimental Techniques for Imaging and Measuring Transient Vapor Nanobubbles. Appl. Phys. Lett. 2012, 101, 264102.

19. Siems, A.; Weber, S. A. L.; Boneberg, J.; Plech, A. Thermodynamics of Nanosecond Nanobubble Formation at Laser-Excited Metal Nanoparticles. New J. Phys. 2011, 13, 043018.

20. Lukianova-Hleb, E. Y.; Lapotko, D. O. Influence of Transient Environmental Photothermal Effects on Optical Scattering by Gold Nanoparticles. Nano Lett. 2009, 9, 2160-2166.

21. Katayama, T.; Setoura, K.; Werner, D.; Miyasaka, H.; Hashimoto, S. Picosecond-to-Nanosecond Dynamics of Plasmonic Nanobubbles from Pump Probe Spectral Measurements of Aqueous Colloidal Gold Nanoparticles. Langmuir 2014, 30, 9504-9513.

22. Lukianova-Hleb, E. Y.; Volkov, A. N.; Lapotko, D. O. Laser Pulse Duration is Critical for the Generation of Plasmonic Nanobubbles. Langmuir 2015, 30, 7425-7434.

23. Hashimoto, S.; Werner, D.; Uwada, T. Studies on the Interaction of Pulsed Lasers with Plasmonic Gold Nanoparticles toward Light Manipulation, Heat Management, and Nanofabrication. J. Photochem. Photobiol. C 2012, 13, 28-54.

24. Baffou, G.; Rigneault, H. Femtosecond-Pulsed Optical Heating of Gold Nanoparticles. Phys. Rev. B 2011, $84,035415$.

25. Huang, W.; Qian, W.; El-Sayed, M. A.; Ding, Y.; Wang, Z. L. Effect of Lattice Crystallinity on the Electron-Phonon Relaxation Rates in Gold Nanoparticles. J. Phys. Chem. C 2007, 111, 10751.

26. Hodak, J. H.; Henglein, A.; Hartland, G. V. Size Dependent Properties of Au Particles: Coherent Excitation and Dephasing of Acoustic Vibrational Modes. J. Chem. Phys. 1999, 111, 8613. 
27. Link, S.; Burda, C.; Wang, Z. L.; El-Sayed, M. A. Electron Dynamics in Gold and Gold-Silver Alloy Nanoparticles: the Influence of a Nonequilibrium Electron Distribution and the Size Dependence of the Electron-Phonon Relaxation. J. Chem. Phys. 1999, 111, 1255.

28. Arbouet, A.; Voisin, C.; Christofilos, D.; Langot, P.; Del Fatti, N.; Vallée, F.; Lermé, J.; Celep, G.; Cottancin, E.; Gaudry, M. et al. Electron-Phonon Scattering in Metal Clusters. Phys. Rev. Lett. 2003, $90,177401$.

29. Hodak, J. H.; Henglein, A.; Hartland, G. V. Electron-Phonon Coupling Dynamics in Very Small (between 2 and 8 nm Diameter) Au Nanoparticles. J. Chem. Phys. 2000, 112, 5942.

30. Letfullin, R. R.; George, T. F.; Duree, G. C.; Bollinger, B. M. Ultrashort Laser Pulse Heating of Nanoparticles: Comparison of Theoretical Approaches. Adv. Opt. Technol. 2008, 2008, 251718.

31. Berto, P.; Mohamed, M. S. A.; Rigneault, H.; Baffou, G. Time-Harmonic Optical Heating of Plasmonic Nanoparticles. Phys. Rev. B 2014, 90, 035439.

32. Schmidt, A. J.; Alper, J. D.; Chiesa, M.; Chen, G.; Das, S. K.; Hamad-Schifferli, K. Probing the Gold Nanorod-Ligand-Solvent Interface by Plasmonic Absorption and Thermal Decay. J. Phys. Chem. C 2008, $112,13320$.

33. Alper, J.; Hamad-Schifferli, K. Effect of Ligands on Thermal Dissipation from Gold Nanorods. Langmuir 2010, 26, 3786.

34. Ge, Z.; Cahill, D. G.; Braun, P. V. Thermal Conductance of Hydrophilic and Hydrophobic Interfaces. Phys. Rev. Lett. 2006, 96, 186101.

35. Baffou, G.; Quidant, R.; Girard, C. Thermoplasmonics Modeling: a Green's Function Approach. Phys. Rev. B 2010, 82, 165424.

36. Löwen, H.; Madden, P. A. A Microscopic Mechanism for Shock-Wave Generation in Pulsed-LaserHeated Colloidal Suspensions. J. Chem. Phys. 1992, 97.

37. Caupin, F.; Herbert, E. Cavitation in Water: A Review. C. R. Physique 2006, 7, 1000 - 1017. 
38. Lombard, J.; Biben, T.; Merabia, S. Kinetics of Nanobubble Generation around Overheated Nanoparticles. Phys. Rev. Lett. 2014, 112, 105701.

39. Lombard, J.; Biben, T.; Merabia, S. Nanobubbles around Plasmonic Nanoparticles: Thermodynamic Analysis. Phys. Rev. E 2015, 91, 043007.

40. Boulais, E.; Lachaine, R.; Meunier, M. Plasma Mediated off-Resonance Plasmonic Enhanced Ultrafast Laser-Induced Nanocavitation. Nano Lett. 2012, 12, 4763-4769.

41. Lachaine, R.; Boulais, E.; Meunier, M. From Thermo- to Plasma-Mediated Ultrafast Laser-Induced Plasmonic Nanobubbles. ACS Photonics 2014, 1, 331-336.

42. Cavicchi, R. E.; Meier, D. C.; Presser, C.; Prabhu, V. M.; Guha, S. Single Laser Pulse Effects on Suspended-Au-Nanoparticle Size Distributions and Morphology. J. Phys. Chem. C 2013, 117, 1086610875 .

43. Carlson, M. T.; Khan, A.; Richardson, H. H. Local Temperature Determination of Optically Excited Nanoparticles and Nanodots. Nano Lett. 2011, 11, 1061-1069.

44. Bohren, C. F.; Huffman, D. R. Absorption and Scattering of Light by Small Particles; Wiley interscience, 1983. 


\section{Graphical TOC Entry}

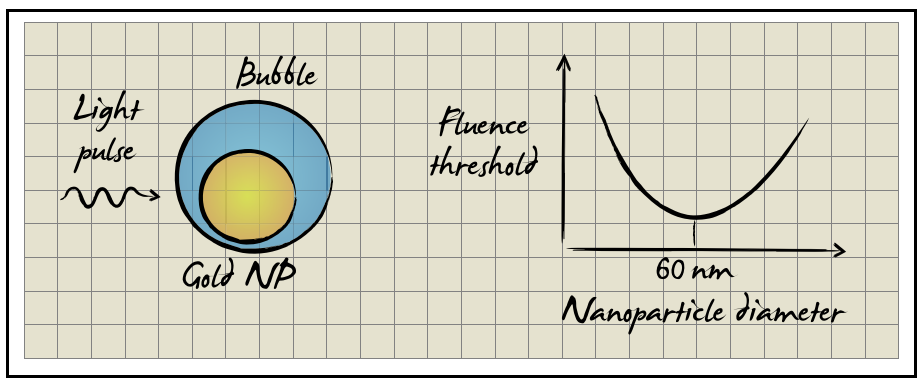

14

15

16

17

18

19

20

21

22

23

24

25

26

27

28

29

30

31

32

33

34

35

36

37

38

39

40

41

42

43

44

45

46

47

48

49

50

51

52

53

54

55

56

57

58

59

60 